\title{
Combined Cytogenetic and Molecular Analyses for the Diagnosis of Prader-Willi/Angelman Syndromes
}

\author{
Daniel Borelina $a^{\dagger}$, Nora Engel ${ }^{\dagger}$, Sebastian Esperante ${ }^{\dagger}$, Veronica Ferreiro ${ }^{\dagger}$, Marcela Ferrer ${ }^{\ddagger}$, \\ Maria Torrado ${ }^{\S}$, Ernesto Goldschmidt ${ }^{\S}$, Liliana Francipane ${ }^{\S}$ and Irene Szijan ${ }^{\dagger, *}$ \\ ${ }^{\dagger}$ Genetica y Biologia Molecular, Facultad de Farmacia y Bioquimica, UBA, Buenos Aires, Argentina \\ Instituto de Neurociencias Aplicadas, UBA, Buenos Aires, Argentina \\ ${ }^{\S}$ Division Genetica, Hospital Jose de San Martin, Buenos Aires, Argentina
}

Received 27 November 2003, Accepted 13 January 2004

\begin{abstract}
Prader-Willi (PWS) and Angelman (AS) are syndromes of developmental impairment that result from the loss of expression of imprinted genes in the paternal (PWS) or maternal (AS) 15q11-q13 chromosome. Diagnosis on a clinical basis is difficult in newborns and young infants; thus, a suitable molecular test capable of revealing chromosomal abnormalities is required. We used a variety of cytogenetic and molecular approaches, such as, chromosome $\mathbf{G}$ banding, fluorescent in situ hybridization, a DNA methylation test, and a set of chromosome 15 DNA polymorphisms to characterize a cohort of 27 PWS patients and 24 suspected AS patients. Molecular analysis enabled the reliable diagnosis of 14 PWS and 7 AS patients, and their classification into four groups: (A) 6 of these 14 PWS subjects $(44 \%)$ had deletions of paternal 15q11-q13; (B) 4 of the 7 AS patients had deletions of maternal 15q11-q13; (C) one PWS patient (8\%) had a maternal uniparental disomy (UPD) of chromosome 15; (D) the remaining reliably diagnoses of 7 PWS and 3 AS cases showed abnormal methylation patterns of 15q11-q13 chromosome, but none of the alterations shown by the above groups, although they may have harbored deletions undetected by the markers used. This study highlights the importance of using a combination of cytogenetic and molecular tests for a reliable diagnosis of PWS or AS, and for the identification of genetic alterations.
\end{abstract}

Keywords: Deletions, Methylation test, Prader-Willi/ Angelman syndromes, Uniparental disomy

*To whom correspondence should be addressed.

Tel: 5411-4964-8296; Fax: 5411-4508-3645

E-mail: iszijan@ffyb.uba.ar; iszijan@arnet.com.ar

\section{Introduction}

PWS and AS syndromes are complex devastating disorders, which are associated with developmental deficiency; they both have prevalences of $c a$. 1:15,000. PWS is associated with mental retardation, severe neonatal hypotonia, and feeding difficulties, which are followed by hyperphagia, obesity, and hypogonadism. AS patients also have feeding difficulties, and ataxia, hyperactivity, seizures, and severe mental retardation (Gillessen-Kaesbach et al., 1995).

These behavioral disorders result from the lack of expression of imprinted genes in the paternal (PWS) or the maternal (AS) 15 chromosome, through different mechanisms. The three most common mechanisms are the deletion of 15q11-q13, uniparental disomy (UPD), and imprinting mutations. Interstitial deletions of the proximal region of paternal 15q have been reported in about $70 \%$ of PWS patients, whereas maternal UPD has been found in $20-25 \%$. AS is associated with maternally derived deletions of $15 \mathrm{q} 11$ q13 (70\%), paternal UPD (2\%), or mutations in the UBE3A gene (20\%) (Nicholls et al., 1998).

Deletions occurring in PWS/AS patients are commonly large and some can be detected by cytogenetic techniques. However, most deletions are detected only by molecular assays. The centromeric deletion breakpoint is frequently located in two regions defined by microsatellites, proximal or distal to D15S542, which have been used to define two types of patients showing deletions, as class I and class II, respectively (Christian et al., 1995).

The main mechanism responsible for the formation of maternal UPD 15 is likely to involve trisomic rescue of trisomic conception with the loss of paternal chromosome 15 (Roberts et al., 1997). In some cases, trisomy may persist but it is rare in live-born infants and it is the most frequent cause for first trimester spontaneous abortion (Kuller and Laifer, 1991). However, mosaic trisomy 15 has been reported in live- 
born infants (Zaslav et al., 1998).

In this study, a group of patients with clinical profiles of PWS or AS was extensively investigated in order to identify the molecular abnormalities associated with these syndromes.

\section{Materials and Methods}

Patients Clinical evaluations were carried out according to the diagnostic criteria for PWS and AS patients (Gillessen-Kaesbach $e t$ al., 1995). These two syndromes present different clinical pictures, which both include mental retardation.

Severe hypotonia and feeding difficulties during the neonatal period are typical features of PWS, which is followed by retarded motor/mental development and behavioral disorders during childhood. The physical appearance of PWS patients involves; characteristic facies, small hands and feet, and a stature below normal. Obesity appears during later childhood-due to greater appetite - and hypogenitalism. Hypopigmentation was observed in patients with a deletion (Holm et al., 1993). The main symptoms of AS patients were feeding difficulties in the neonatal period, developmental delay, microcephaly, severe mental retardation, absence of speech, hyperactivity, ataxia, seizures, a happy appearance and hypopigmentation (Williams et al., 1995). PWS and AS phenotypes are variable, and clinical diagnosis is difficult in newborns and young infants. In the present study, the subjects' ages at diagnosis ranged from 1 to 13 yr. Only one was diagnosed earlier (at $1 \mathrm{mo}$ ) owing to severe malnutrition. All of the AS patients were diagnosed after $1 \mathrm{yr}$ (Table 1).

Table 1. Classification of PWS/AS patients based on an abnormal methylation test

\begin{tabular}{|c|c|c|c|c|c|}
\hline \multirow{2}{*}{ Patients } & \multirow{2}{*}{ Sex/age (yr) } & \multirow{2}{*}{ Karyotype } & \multicolumn{3}{|c|}{ Method } \\
\hline & & & Cytogenetic & FISH & Molecular \\
\hline \multicolumn{6}{|c|}{ Group A: PWS, paternal deletion in the PWS/AS critical region } \\
\hline PWS1 & $\mathrm{F} / 13$ & $46, X X, \operatorname{del}(15)^{1}$ & - & + & + \\
\hline PWS3 & $\mathrm{F} / 1 \mathrm{mo}$ & $46, X, \operatorname{del}(15 q)^{2}$ & + & + & + \\
\hline PWS5 & $\mathrm{M} / 19 \mathrm{mo}$ & $46, \mathrm{XY}^{*}$ & - & - & + \\
\hline PWS8 & $\mathrm{F} / 3$ & $46, \mathrm{XX}, \operatorname{del}(15 q)^{2}$ & + & + & + \\
\hline PWS9 & $\mathrm{M} / 6$ & $46, X Y, \operatorname{del}(15 q)^{1}$ & - & + & + \\
\hline PWS11 & $\mathrm{F} / 9$ & $46, \mathrm{XX}, \operatorname{del}(15 \mathrm{q})^{1}$ & + & + & \\
\hline \multicolumn{6}{|c|}{ Group B:AS, maternal deletion in the PWS/AS critical region } \\
\hline AS2 & $\mathrm{M} / 5$ & $46, X Y, \operatorname{del}(15 q)^{3}$ & + & + & + \\
\hline AS4 & $\mathrm{F} / 5$ & ND* & & & + \\
\hline AS5 & $\mathrm{M} / 10$ & ND* & & & + \\
\hline AS7 & $\mathrm{M} / 6$ & ND* & & & + \\
\hline \multicolumn{6}{|c|}{ Group C: maternal UPD(15) with SMC(15) } \\
\hline PWS7 & $\mathrm{F} / 3$ & $46, \mathrm{XX}^{4}$ & - & - & + \\
\hline \multicolumn{6}{|c|}{ Group D: molecular alteration not determined (deletion or UPD or other) } \\
\hline PWS2 & $\mathrm{F} / 5$ & $46, \mathrm{XX}^{4}$ & - & $-\mathrm{ND}$ & \\
\hline PWS4 & $\mathrm{M} / 6$ & $46, X Y^{5}$ & - & - & ND \\
\hline PWS6 & $\mathrm{M} / 8$ & $46, X Y^{5}$ & - & - & ND \\
\hline PWS10 & $\mathrm{M} / 7$ & ND & & & \\
\hline PWS12 & $\mathrm{F} / 10$ & $46, X^{5}$ & - & ND & \\
\hline PWS13 & $\mathrm{F} / 6$ & ND & & & \\
\hline PWS14 & $\mathrm{M} / 5$ & ND & & & \\
\hline AS1 & $\mathrm{M} / 6$ & ND & & & \\
\hline AS3 & $\mathrm{M} / 7$ & ND & & & \\
\hline \multirow[t]{4}{*}{ AS6 } & $\mathrm{F} / 6$ & ND & & & \\
\hline & & Total PWS deletions & $2(18 \%)$ & $5(46 \%)$ & $6(100 \%)$ \\
\hline & & Total PWS UPD & 0 & 0 & 1 \\
\hline & & Total AS deletions & 1 & 1 & 4 \\
\hline
\end{tabular}

F, female; M, male; ND, not determined; UPD, uniparental disomy; *, deletion detected by microsatellite analysis

1, deletion detected by FISH; 2, deletion detected by cytogenetic and FISH; 3, cytogenetic analysis; 4, normal karyotype and FISH; 5, normal karyotype. 
Cytogenetic studies Peripheral blood lymphocytes were cultured using standard methods (Yunis, 1976). Complete karyotype analysis of G-banded chromosomes (trypsin-Giemsa staining using standard techniques) was performed, and attention was paid to the possibility of a chromosome 15 abnormality.

Fluorescence in situ hybridization (FISH) study on metaphase spreads was performed with probes for chromosome 15 markers, SNRPN (15q11-q13 region), D15Z1 (15p11.2), and PML (15q22) (Abbott Vysis, Chicago USA). FISH was carried out according to the manufacturers instructions.

\section{Molecular studies}

DNA methylation analysis

Southern blot: DNA from peripheral blood was digested with Hind III and Hpa II, electrophoresed in 1\% agarose gels and analyzed by hybridization using PW71 as probe (D15S63 locus for the SNRPN gene). The probe was labeled with alpha ${ }^{32} \mathrm{P}-\mathrm{dCTP}$ by random oligonucleotide priming. Post-hybridization washes and result interpretations were carried out according to Dittrich et al. (1992). Normal individuals have a maternally derived band at $6.0 \mathrm{~Kb}$ and a paternally derived band at $4.4 \mathrm{~Kb}$.

Methylation-specific PCR (M-PCR) was performed using DNA previously treated with sodium bisulfite, which converts cytocine to uracil except when the cytosine is methylated. PCR primers specific for the methylated and unmethylated $\mathrm{CpG}$ island of SNRPN were designed by Kubota et al. (1997), and the M-PCR amplification technique used was as described by the same authors (Kubota et al., 1997). Normal individuals show a maternally derived 174 bp PCRproduct and a paternally derived 100-bp band.

\section{Microsatellite assay}

CA dinucleotide repeat polymorphisms are ideal for familial studies (Dalamon et al., 2003; Giliberto et al., 2003), because they are multiallelic and have high polymorphic information content (>70\%). Five chromosome 15 polymorphic loci were used: D15S542 (Christian et al., 1995), A55CA-1 (Glatt, Sinnett and Lalande, 1994), D15S97, D15S131, and D15S211 (Beckmann et al., 1993). The A55CA-1 and D15S97 microsatellites showed the parental origin of the PWS/AS critical region. UPD can be detected by the presence of 2 alleles from one parent, which are not shared with the other parent. The origin of nondisjunction can be established using the centromeric microsatellites D15S542 or A55CA-1, located genetically close to the centromere, and thus, are representative of centromeric status with a $90 \%$ probability (Mutirangura et al., 1993). The heterodisomic state of these polymorphic markers indicates a Meiosis I error, and their isodisomy indicates a nondisjunction in the Meiosis II stage or a postzygotic event (Robinson et al., 1993).

\section{Results}

Twenty-seven patients presented clinical symptoms of PWS and 24 suspected of having AS were studied by cytogenetic and molecular methods. An abnormal SNRPN pattern, with no paternal contribution, was detected in 14 PWS patients, and an abnormal SNRPN pattern with a lack of maternal
Table 2. Polymorphisms studies in selected patients

\begin{tabular}{|c|c|c|c|}
\hline & D15S542 & A55CA-1 & D15S131 \\
\hline \multicolumn{4}{|c|}{ Group A: PWS, paternal deletion } \\
\hline M & 1,5 & 4,4 & 2,3 \\
\hline $\mathrm{P}$ & 2,5 & 4 & 2.3 \\
\hline $\mathrm{F}$ & 2,3 & 1,3 & 1,3 \\
\hline \multicolumn{4}{|c|}{ Group B: AS, maternal deletion } \\
\hline M & 1,3 & 3,5 & 1,5 \\
\hline $\mathrm{P}$ & 3 & 4 & 3,5 \\
\hline $\mathrm{F}$ & 3,5 & 1,4 & 2,3 \\
\hline \multicolumn{4}{|l|}{ AS5 } \\
\hline M & 2,3 & 1,2 & 2,3 \\
\hline $\mathrm{P}$ & 1,3 & 3 & 3,4 \\
\hline $\mathrm{F}$ & 1,1 & 2,3 & 1,4 \\
\hline \multicolumn{4}{|l|}{ AS7 } \\
\hline M & 1,2 & 3,4 & 1,2 \\
\hline $\mathrm{P}$ & 3 & 1 & 2,3 \\
\hline $\mathrm{F}$ & 1,3 & 1,2 & 3,4 \\
\hline \multicolumn{4}{|c|}{ Group C: PWS, maternal UPD (15) } \\
\hline M & 1,3 & 2,3 & 1,2 \\
\hline $\mathrm{P}$ & 1,3 & 2,3 & 1,2 \\
\hline $\mathrm{F}$ & 2,3 & 1,4 & 1,3 \\
\hline
\end{tabular}

M, mother; F, father; P, proband; UPD, uniparental disomy. Two additional polymorphisms, D15S97 and D15S211, were tested in families PWS5 and PWS14. D15S97 alleles in family PWS5 were: M, 1,4; P, 4; F, 2,3, D15S211 was uninformative. None of the two polymorphisms was informative in family PWS14.

contribution in 7 AS cases. These data emphasize the importance of molecular testing for PWS/AS diagnosis due to the difficulty of establishing a clear clinical picture. Patients with reliable molecular findings of PWS or AS were classified according to their clinical, cytogenetic, and molecular abnormalities (Table 1).

Deletions in the PWS/AS critical region The methylation test can detect deletions, UPD, and mutations in the imprinting center of PWS/AS patients, but cannot differentiate between these alterations. Thus, gross rearrangements were identified by cytogenetic and microsatellite analyses (Tables 1 and 2). Deletions involving the paternal copy of the PWS/AS region were detected in 6 PWS patients (Group A), 2 by cytogenetic $(2 / 11=18 \%), 5$ by FISH $(5 / 11=46 \%)$ and 6 by microsatellite $(6 / 6=100 \%)$ techniques. Four of the 6 PWS deletions were submicroscopic and hence, not detectable by cytogenetic testing (PWS 1, 5, 9, and 11). They were detected by FISH and microsatellite analyses (Fig. 1 shows a FISH picture of PWS8). Deletions, including the maternal copy of the PWS/AS region, were detected in 4 AS subjects (Group 


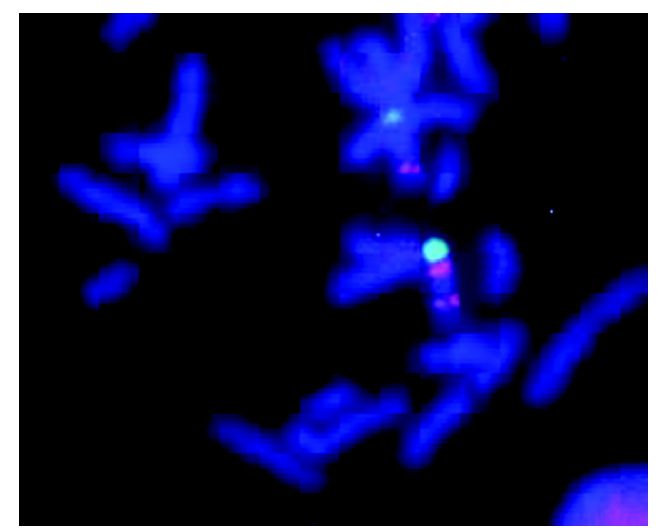

Fig. 1. Fluorescence in situ hybridization (FISH) assay. The DNA of patient PWS8 revealed alpha satellite D15Z1 and PML signals on both 15 chromosomes, and signal of the SNRPN probe in only one of these chromosomes.

B) one by cytogenetic techniques and FISH and 4 by microsatellite techniques. Three polymorphic loci were assessed, resulting informative one or two in each family. Additional markers, i.e., D15S97 and D15S211, were used in two cases, and produced informative results in one case (D15S97, Table 2). AS case 2 with a cytogenetically detected deletion, presented an atypical appearance, due to dark skin and hair

Maternal UPD(15) PW7 showed the classical clinical features of PWS at age three. The methylation test of DNA showed an abnormal SNRPN pattern with no paternal contribution (data not shown). Segregation analysis using DNA polymorphisms, mapping throughout chromosome 15 documented two maternal alleles but no paternal allele at the PWS/AS locus (A55AC-1) (Table 2). Heterozygosity at the PWS critical region excludes the possibility of deletion, furthermore, the subject showed the same heterozygous pattern as her mother at other loci. These results are consistent with a maternal uniparental heterodisomy of chromosome 15 .

This genetic aberration was not determined in subjects with an altered 15q11-q13 methylation pattern.

No molecular abnormality could be characterized in 7 PWS or in 3 AS patients with an abnormal methylation at the PWS/ AS critical region (Group D). Four of the PWS subjects studied by chromosomal analysis showed a normal karyotype and one was normal by FISH. The parents of these patients were unavailable for a polymorphism study. PWS case 12 with a normal karyotype had a mild phenotype.

Patients with no detected molecular abnormality Thirteen patients suspected of having PWS and 17 presumptive AS patients showed a normal methylation pattern. Several were studied by microsatellite segregation analysis using DNA from lymphocytes and other tissue, buccal mucosa (data not shown), in order to detect possible tissue mosaicism for the genetic alteration. All showed biparental inheritance and were heterozygous at the PWS/AS locus. Some of these patients displayed all the clinical features of classical PWS or AS, but others only presented the more typical symptoms like hypotonia and developmental delay. These features are common to other genetic disorders such as metabolic inborn errors, the majority present with hypotonia and a failure to thrive (Burton, 1998).

One AS subject was a familial case, there were two affected siblings; however, the methylation pattern of the PWS/AS region showed no alterations, suggesting point mutations in the UBE3A gene.

\section{Discussion}

Cytogenetic and detailed molecular studies of patients with the clinical symptoms of PWS or AS, using probes for the PWS/AS critical region and chromosome 15 polymorphisms allowed reliable diagnosis and revealed molecular alterations. Ten genetic abnormalities were detected; deletions (6 PWS and 4 AS) and one PWS patient harbored maternal UPD. In two of patients studied by microsatellite analysis (PWS5 and AS5) the deletion involves markers at D15S97 and at A55CA1 (PWS5) or at A55CA-1 (AS5), but not at D15S542. On the other hand, in subject AS7, and probably in AS4 (because of homozygosity) the deletion breakpoint was centromeric to this polymorphism. Christian et al. (1995) used the polymorphic marker D15S542 to characterize the centromeric common deletion breakpoint region. These authors defined two classes of PWS and AS patients, approximately 50\% were deleted (class I) and 50\% were not deleted (class II). Thus, in our study PWS5 and AS5 were class II patients, whereas AS7 and probably AS4 were class I.

PWS7 showed maternal uniparental heterodisomy, heterozygosity for maternal alleles at a locus close to the centromere suggests (with 90\% likelihood) that the nondisjunction is a Meiosis I error, since the probability of a recombination between this locus and the centromere is low (Mutirangura et al., 1993).

Half of the patients with PWS and most of the suspected AS subjects produced normal methylation results and paternal/ maternal inheritance of chromosome 15 microsatellites. Some of these patients did not fulfill the diagnostic criteria for PWS or AS, though they had some typical features, such as, hypotonia and developmental delay. However, other patients with no detected molecular abnormality were also evaluated by experienced clinicians as having PWS or AS. These patients may have harbored other alterations, such as, small mutations in candidate PWS (SNRPN) or AS (UBE3A) genes, which would not have been detected by the methylation and microsatellite testing. Point mutations in UBE3A have been identified in $20 \%$ of AS subjects, whereas no PWS subjects have been reported to have small mutations in SNRPN or other PWS candidate genes (Nicholls, Saitoh 
and Horsthemke, 1998). Limited deletions - undetectable by the markers used-may be the cause for PWS in some patients. However, the majority of patients with clinical PWS or AS were probably misdiagnosed, because classical PWS and AS symptoms are common to many diseases (Burton, 1998).

This study emphasizes the importance of molecular analyses for: 1) the reliable diagnosis of Prader-Willi and Angelman syndromes, which share clinical symptoms with many other disorders; and 2) for the determination of the mechanisms of genetic alteration, thus allowing us to better understand the role of imprinting in genetic diseases. The analysis of the extents of deletions may provide an insight into identifying the genes involved in these syndromes.

Acknowledgments The authors are grateful to the patients' families for their collaboration. This work was supported by grants from the Universidad de Buenos Aires (B069) and from the Agencia Nacional de Promoción Científica y Tecnológica (BID 1201/OC-AR-PICT 5-8684).

\section{References}

Beckmann, J. S., Tomfohrde, J., Barnes, R. I., Williams, M., Broux, O., Richard, I. Weissenbach, J. and Bowcock, A. M. (1993) A linkage map of human chromosome 15 with an average resolution of $2 \mathrm{cM}$ and containing 55 polymorphic microsatellites. Hum. Mol. Genet. 2, 2019-2030.

Burton, B. K. (1998) Inborn errors of metabolism: a guide to diagnosis. Pediatrics 102, 1-9.

Christian, S. L., Robinson, W. P., Huang, B., Mutirangura, A., Line, M. R., Nakao, M., Surti, U., Chakravarti, A. and Ledbetter, D. H. (1995) Molecular characterization of two proximal deletion breakpoint regions in both Prader-Willi and Angelman syndrome patients. Am. J. Hum. Genet. 57, 40-48.

Dalamon, V., Surace, E., Giliberto, F., Ferreiro, V., Fernández, C. and Szijan, I. (2003) Detection of germline mutations in Argentine retinoblastoma patients. Low and full penetrance retinoblastoma caused by the same germline mutation. $J$. Biochem. Mol. Biol. 36, 179-184

Dittrich, B., Robinson, W. P., Knoblauch, H., Buiting, K., Schmidt, K., Gillesen-Kaesbach, G. and Horsthemke, B. (1992) Molecular diagnosis of the Prader-Willi and Angelman syndromes by detection of parent-of-origin specific DNA methylation in 15q11-13. Hum. Genet. 90, 313-315.

Giliberto, F., Ferreiro, V., Dalamon, V., Surace, E., Cotignola, J., Esperante, S., Borelina, D., Baranzini, S. and Szijan, I. (2003)
Direct deletion analysis in two Duchenne muscular dystrophy symptomatic females using polymorphic dinucleotide (CA) $)_{n}$ loci within the dystrophin gene. J. Biochem. Mol. Biol. 36, 179-184.

Gillessen-Kaesbach, G., Robinson, W., Dietmar, L., KayaWesterloh, S., Passarge, E. and Horsthemke, B. (1995) Genotype-phenotype correlation in a series of 167 deletion nondeletion patients with Prader-Willi syndrome. Hum. Genet. 96, 638-664.

Glatt, K., Sinnett, D. and Lalalnde, M. (1994) The human $\gamma$ aminobutyric acid receptor subunit $\beta 3$ and $\alpha 5$ gene cluster in chromosome $15 \mathrm{q} 11-\mathrm{q} 13$ is rich in highly polymorphic (CA) repeats. Genomics 19, 157-160.

Holm, V. A., Cassidy, S. B., Butler, M. G., Hanchet, J. M., Greenswag, L. R., Whitman, B. Y. and Greeenberg, F. (1993) Prader-Willi syndrome consensus diagnostic criteria. Pediatrics 91, 398-402.

Kubota, T., Das, S., Christian, S. L., Baylin, S. B., Herman, J. G. and Ledbetter, D. H. (1997) Methylation-specific PCR simplifies imprinting analysis. Nat. Genet. 16, 16-17.

Kuller, J. A. and Laifer, S. A. (1991) Trisomy 15 associated with nonimmune hydrops. Am. J. Perinatol. 8, 39-40.

Mutirangura, A., Greenberg, F., Butler, M. G., Malcolm, S., Nicholls, R. D., Chakravarti, A. and Ledbetter, D. H. (1993) Multiplex PCR of three dinucleotide repeats in the PraderWilli/Angelman critical region (15q11-q13): Molecular diagnosis and mechanism of uniparental disomy. Hum. Mol. Genet. 2, 143-151.

Nicholls, R. D., Saitoh, S. and Horsthemke, B. (1998) Imprinting in Prader-Willi and Angelman syndromes. Trends Genet.14, 194-200.

Roberts, E., Stevenson, K., Cole, T., Redford, D. H. and Davison, E. V. (1997) Prospective prenatal diagnosis of Prader-Willi syndrome due to maternal disomy for chromosome 15 following trisomy zygote rescue. Prenat. Diagn. 17, 780-783.

Robinson, W. P., Bernasconi, F., Mutirangura, A., Ledbetter, D. H., Langlois, S., Malcolm, S., Morris, M. A. and Schinzel, A. A. (1993) Nondisjunction of chromosome 15: Origin and recombination. Am. J. Hum. Genet. 53, 740-751.

Williams, C. A., Angelman, H., Clayton-Smith, J., Driscoll, D. J., Hendrickson, J. E., Knoll, J. H. M., Magenis, R. E., Schinzel, A., Wagstaff, J., Whidden, E. M. and Zori, R. T. (1995) Angelman syndrome: Consensus for diagnostic criteria. Am. J. Med. Genet. 56, 237-238.

Yunis, J. J. (1976) High resolution of human chromosomes. Science 191, 1268-1270.

Zaslav, A. L., Fallet, S., Brown, S., Ebert, R., Fleischer, A., Valderama, E. and Fox, J. E. (1998) Prenatal diagnosis of low level trisomy 15 mosaicism: Review of the literature. Clin. Genet. 53, 286-292. 